\title{
Factors Affecting Quality of Life and Satisfaction in Patients with Arthritis after Change to a Fixed- Dose Naproxen/Esomeprazole Combination Drug
}

\author{
Daehyun Park, MD, Yonguk Kwon, MD \\ Department of Orthopedics, Inje University Busan Paik Hospital, Busan, Korea
}

Background: In drug therapy for patients with arthritis, a naproxen/esomeprazole combination drug may be a tolerable choice because it can minimize gastrointestinal and cardiovascular adverse effects. The aim of this study was to investigate the changes in quality of life (0OL), medication adherence, and satisfaction after switch from the existing drug to the combination drug. In addition, we analyzed the correlation between the above-mentioned variables and the stratified demographic and medical data of the patients.

Methods: A prospective, noninterventional, observational study was conducted in 30 hospitals between May 2014 and July 2016. In total, 2,308 patients with osteoarthritis, 99 patients with rheumatoid arthritis, and 76 patients with ankylosing spondylitis were enrolled. Demographic information (age, sex, body mass index [BMI], alcohol consumption, and smoking) and medical information (type of arthritis, duration of disease, and comorbidities) were collected via a self-administered questionnaire. Patients were observed for more than three months after switching to the combination drug. Data on the 0OL (EuroQoL 5-Dimension questionnaire [EQ-5D questionnaire]), medication adherence (Morisky Medication Adherence Scale [MMAS]), and satisfaction were collected at the first and last visits.

Results: A total of 2,483 patients enrolled at 30 hospitals completed the questionnaire. After the switch to the combination drug, the mean EQ-5D score improved from $0.72 \pm 0.17$ to $0.79 \pm 0.14(p<0.001)$, and significant improvement was associated with female sex $(p=0.016)$, shorter disease duration $(p<0.001)$, and absence of comorbidities $(p<0.001)$. The mean MMAS score was 6.38 \pm 1.77 , indicating medium adherence. Satisfaction was significantly higher in female patients $(p<0.001)$, in patients with a shorter disease duration $(p<0.001)$, osteoarthritis $(p=0.003)$, and no comorbidities $(p<0.001)$. Serious drug-related adverse effects did not occur.

Conclusions: The overall QOL was improved with medium adherence after the switch to the combination drug. On the basis of the analysis of stratified data, sex, age, drinking, smoking, disease duration, comorbidities, and BMI might be associated with QOL, satisfaction, and adherence.

Keywords: Nonsteroidal anti-inflammatory drugs, Arthritis, Naproxen, Quality of life

Received February 13, 2019; Accepted September 9, 2019

Correspondence to: Daehyun Park, MD

Department of Orthopedics, Inje University Busan Paik Hospital, 75 Bokji-

ro, Busanjin-gu, Busan 47392, Korea

Tel: +82-51-890-6257, Fax: +82-51-892-6619

E-mail: spineparkdaehyun@gmail.com
Nonsteroidal anti-inflammatory drugs (NSAIDs) are the most commonly prescribed drugs worldwide and are mainly indicated in osteoarthritis, rheumatoid arthritis, and musculoskeletal pain. ${ }^{1)}$ In the United States, at least $70 \%$ of people aged 65 years or older take NSAIDs at a minimum of once a week and as the elderly population increases, the use of the medication increases rapidly. ${ }^{2)}$ Several studies have shown that a single agent of NSAID 
has no definite superior effects over the other types; moreover, it is important to select an appropriate NSAID to minimize the adverse effects and to improve the quality of

\section{Table 1. Demographic Data}

\begin{tabular}{|c|c|}
\hline Variable & No. of cases $(\%)$ \\
\hline \multicolumn{2}{|l|}{ Age (yr) } \\
\hline 19-29 & $31(1.3)$ \\
\hline 30-39 & $73(2.9)$ \\
\hline $40-49$ & $191(7.7)$ \\
\hline $50-59$ & $487(19.6)$ \\
\hline $60-69$ & $813(32.7)$ \\
\hline$\geq 70$ & 888 (35.8) \\
\hline \multicolumn{2}{|l|}{ Sex } \\
\hline Male & $1,177(47.4)$ \\
\hline Female & $1,306(52.6)$ \\
\hline \multicolumn{2}{|l|}{ Smoking } \\
\hline Current smoker & $351(14.1)$ \\
\hline Ex-smoker & $274(11.0)$ \\
\hline Non-smoker & $1,744(70.3)$ \\
\hline Unknown & $114(4.6)$ \\
\hline \multicolumn{2}{|l|}{ Alcohol drinking } \\
\hline Current drinker & 665 (26.8) \\
\hline Ex-drinker & $233(9.4)$ \\
\hline Non-drinker & 1,476 (59.4) \\
\hline Unknown & $109(4.4)$ \\
\hline
\end{tabular}

life (QOL). ${ }^{3)}$ Therefore, most domestic and global NSAID prescription guidelines are focused on minimizing drugrelated adverse effects. ${ }^{4,5)}$

The most common side effects of NSAIDs are gastrointestinal (GI) complications (dyspepsia, bleeding, ulcers, and perforation) and cardiovascular (CV) complications (thrombotic events, myocardial infarction, and stroke). Gastroprotective agents such as H2 blockers or proton pump inhibitors (PPIs) are recommended to reduce GI complications; however, patient adherence might be poor. Selective cyclooxygenase (COX)-2 inhibitors have demonstrated reduced GI complications, but not with long-term use, and concerns regarding CV complications have been raised. Among traditional NSAIDs, naproxen (1,000 mg/day) has not been associated with an increased risk for CV events. ${ }^{6)}$ Therefore, a combination of naproxen and PPIs are recommended in order to minimize GI and CV complications. ${ }^{7}$

The choice of NSAID should be tailored individually. Several factors should be considered when prescribing NSAIDs such as age, sex, duration of disease, comorbidities, medication history, and history of GI or CV disease. The final goal is to improve the overall QOL and satisfaction. Health-related QOL outcome are important for evaluating the impact of medical treatment on chronic arthritis. It is well recognized that marked improvements in health-related QOL occur when patients with arthritis take NSAIDs. ${ }^{8,9)}$ GI events are a major problem with NSAIDs and closely related, both directly and indirectly, to negative effects on QOL. Dyspepsia and upper abdominal pain are strongly related to a poor QOL than other symptoms. ${ }^{10)}$

In this study, the QOL and satisfaction were assessed after a change from the existing NSAID therapy to a naproxen/esomeprazole fixed-dose combination drug

Table 2. Quality of Life after Changing to the Combination Drug

\begin{tabular}{lcccccc}
\multicolumn{1}{c}{ Variable } & Number & Enrollment & Last visit & Change & $p$-value \\
\hline Ineffectiveness & 1,729 & $0.71 \pm 0.16$ & $0.79 \pm 0.14$ & $0.07 \pm 0.15$ & $<0.001$ \\
\hline Gl disorder & 233 & $0.72 \pm 0.21$ & $0.81 \pm 0.13$ & $0.09 \pm 0.21$ & $<0.001$ \\
\hline CV risk group & 19 & $0.66 \pm 0.25$ & $0.81 \pm 0.24$ & $0.14 \pm 0.17$ & $0.19 \pm 0.14$ & $<.001$ \\
\hline Cost burden & 20 & $0.60 \pm 0.26$ & $0.79 \pm 0.21$ & $0.05 \pm 0.09$ & $0.08 \pm 0.16$ & $<0.001$ \\
\hline Low compliance & 77 & $0.81 \pm 0.11$ & $0.81 \pm 0.14$ & $0.08 \pm 0.16$ & $<0.001$ \\
\hline Other & 49 & $0.74 \pm 0.17$ & $0.79 \pm 0.14$ & $0.72 \pm 0.17$ & &
\end{tabular}

Values are presented as mean \pm standard deviation.

Gl: gastrointestinal, CV: cardiovascular. 
(combination drug). The demographic data and medical information of the patients were stratified and the correlations between these individual factors and QOL and satisfaction were analyzed. The purpose of this study was to analyze the various factors that can affect QOL and satisfaction to provide information that can be helpful for prescribing NSAIDs for the treatment of arthritis.

\section{METHODS}

This prospective, multicenter, noninterventional, observational study was conducted between May 2014 and July 2016. A total of 30 hospitals were included and each hospital received its own independent Institutional Review Board approval. Written consent from the patients was obtained in accordance with the provision of Korean Good Clinical Practice. All patients were $>19$ years of age and diagnosed as having osteoarthritis, rheumatoid arthritis, or ankylosing spondylitis. The exclusion criteria were hypersensitivity to naproxen and esomeprazole, history of allergic reactions to aspirin or other NSAIDs, active peptic ulcers, and cerebral hemorrhage. The prescription of the medication was made entirely at the discretion of each investigator.

The observation period was more than 3 months and the data were collected via questionnaires at the first

Table 3. Quality of Life Changing by the Stratified Demographic and Medical Information

\begin{tabular}{|c|c|c|c|c|}
\hline Variable & Enrollment & Last visit & Change & $p$-value \\
\hline \multicolumn{5}{|l|}{ Sex } \\
\hline Male & $0.73 \pm 0.15$ & $0.80 \pm 0.14$ & $0.07 \pm 0.14$ & \\
\hline Female & $0.70 \pm 0.18$ & $0.79 \pm 0.14$ & $0.09 \pm 0.17$ & 0.016 \\
\hline \multicolumn{5}{|l|}{ Age (yr) } \\
\hline $19-29$ & $0.79 \pm 0.10$ & $0.84 \pm 0.09$ & $0.05 \pm 0.11$ & \\
\hline $30-39$ & $0.76 \pm 0.17$ & $0.84 \pm 0.15$ & $0.08 \pm 0.14$ & \\
\hline $40-49$ & $0.76 \pm 0.15$ & $0.84 \pm 0.11$ & $0.07 \pm 0.12$ & \\
\hline 50-59 & $0.74 \pm 0.14$ & $0.83 \pm 0.11$ & $0.09 \pm 0.14$ & \\
\hline $60-69$ & $0.72 \pm 0.16$ & $0.79 \pm 0.14$ & $0.07 \pm 0.16$ & \\
\hline$\geq 70$ & $0.69 \pm 0.18$ & $0.76 \pm 0.15$ & $0.08 \pm 0.17$ & \\
\hline \multicolumn{5}{|l|}{ Duration of disease } \\
\hline$\geq$ Mean & $0.70 \pm 0.16$ & $0.76 \pm 0.16$ & $0.05 \pm 0.15$ & \\
\hline$<$ Mean & $0.72 \pm 0.17$ & $0.81 \pm 0.12$ & $0.09 \pm 0.16$ & $<0.001$ \\
\hline \multicolumn{5}{|l|}{ Arthritis type } \\
\hline $\mathrm{OA}$ & $0.71 \pm 0.16$ & $0.79 \pm 0.14$ & $0.08 \pm 0.15$ & \\
\hline RA & $0.72 \pm 0.22$ & $0.82 \pm 0.19$ & $0.10 \pm 0.23$ & \\
\hline AS & $0.78 \pm 0.21$ & $0.84 \pm 0.16$ & $0.06 \pm 0.18$ & \\
\hline \multicolumn{5}{|l|}{ Comorbidity } \\
\hline Yes & $0.71 \pm 0.16$ & $0.76 \pm 0.15$ & $0.06 \pm 0.15$ & \\
\hline No & $0.73 \pm 0.17$ & $0.83 \pm 0.11$ & $0.10 \pm 0.16$ & $<0.001$ \\
\hline \multicolumn{5}{|l|}{ Body mass index } \\
\hline$\geq$ Median & $0.72 \pm 0.16$ & $0.79 \pm 0.14$ & $0.07 \pm 0.14$ & \\
\hline$<$ Median & $0.71 \pm 0.18$ & $0.80 \pm 0.14$ & $0.09 \pm 0.17$ & \\
\hline
\end{tabular}

Values are presented as mean \pm standard deviation.

OA: osteoarthritis, RA: rheumatoid arthritis, AS: ankylosing spondylitis. 
Park and Kwon. Naproxen/Esomeprazole Combination Drug

Clinics in Orthopedic Surgery • Vol. 12, No. 1, $2020 \bullet$ www.ecios.org

and final visits after obtaining written informed consent from the patients. Data on age, sex, body mass index (BMI), alcohol consumption, and smoking status were collected as demographic information; data on the duration of arthritis and other comorbidities were collected as medical information. The QOL was assessed by using an EuroQoL 5-Dimension questionnaire (EQ-5D questionnaire). ${ }^{11}$ Minimal clinically important difference of EQ-5D in this study was 0.08 , which is approximately half a standard deviation. ${ }^{12)}$ Medication adherence was assessed by the Morisky Medication Adherence Scale (MMAS) with scores $<6$ indicating poor adherence, 6 to $<8$ indicating medium adherence, and 8 indicating high performance. ${ }^{13)}$ The drug history prior to enrollment and the reasons for the change were investigated. Satisfactory, unsatisfactory, or no difference were the options to be selected during the evaluation of satisfaction at the end of the observation period. In addition, adverse events that occurred throughout the study period were recorded.

Demographic information, medical information, QOL (EQ-5D), and satisfaction were analyzed by multiple regression and multinomial logistic regression. The statistical program used was SAS ver. 9.4 (SAS institute, Cary, NC, USA).

\section{RESULTS}

A total of 3,650 patients consented to participate in the study. Of these, 2,483 (68\%) completed the study, and the main reasons for not completing the study were loss of follow-up ( $\mathrm{n}=998,27.1 \%)$ and adverse effects after changing the drug $(n=103,2.8 \%)$. The mean age was $64.1 \pm 12.2$ years and the largest age group comprised patients over 70 years. There were 1,306 female (52.6\%) and 1,177 male (47.4\%) patients. The mean BMI was $24.3 \pm 3.32 \mathrm{~kg} / \mathrm{m}^{2}$ (Table 1).

Osteoarthritis was the commonest diagnosis $(\mathrm{n}=$ 2,308, 93.0\%), followed by rheumatoid arthritis $(\mathrm{n}=99$, $3.9 \%)$ and ankylosing spondylitis $(\mathrm{n}=76,3.1 \%)$. The mean disease duration was $42.1 \pm 60.7$ months. There were 1,320 patients (53.2\%) with comorbidities.

NSAIDs were being taken by 2,092 patients (98.4\%) at enrollment and coxibs were the most commonly used drugs. A total of 1,209 patients (56.9\%) were taking gastroprotective agents and $\mathrm{H} 2$ blockers were most commonly used. The commonest reason for changing the drug at enrollment was ineffectiveness of the prior drug in 1,729 patients $(81.3 \%)$, followed by GI disorders in 233 (11.0\%), and risks for CV disease in 19 (0.9\%).

QOL (EQ-5D) significantly improved after switch to the combination drug and the highest improvement was observed when the medication was changed because of cost burden (Table 2). Stratified demographic and medical information were analyzed to investigate the correlation with QOL. The QOL was significantly improved in female patients, those with a shorter disease duration, and those who had no comorbidities (Table 3).

Overall, the mean MMAS score was $6.38 \pm 1.77$ points, indicating medium adherence. The adherence was significantly higher in patients aged 70 years or older, nondrinkers, current smokers, and those with a shorter disease duration, osteoarthritis, comorbidities, and low BMI (Table 4).

A total of 1,338 patients (62.91\%) selected satisfactory and $517(24.31 \%)$ selected no difference on the survey at the last visit. The satisfaction was significantly higher in female patients, those who were aged between 50 and 59 years, were not a current smoker, or had a shorter disease duration, osteoarthritis patients, and those with no comorbidities (Table 5).

A total of 148 patients (5.9\%) experienced adverse events after changing drugs, and 199 adverse events were reported. Dyspepsia was the most common adverse event, occurring in 26 patients (1.05\%), followed by arthralgia in 19 patients $(0.77 \%)$. Eighteen patients $(0.73 \%)$ complained of GI disorders, and dizziness was reported by 17 patients (0.68\%). Permanent withdrawal of the drug was the common treatment for adverse events (103 patients, 51.7\%).

\section{DISCUSSION}

GI complications are the main cause of reduced QOL and discontinuation of the drug. H2 blockers or PPIs are recommended in combination with NSAIDs in many prescription guidelines. PPIs are more effective than $\mathrm{H} 2$ blockers in preventing ulcer lesions and relieving symptoms of heartburn and dyspepsia. ${ }^{14)}$ However, according to a study in Korea, only $41 \%$ of patients took a gastroprotective agent even in the group at a high risk for gastropathy. ${ }^{15)}$ In a retrospective, cohort, observational study of 69,000 patients, only $7.9 \%$ took gastroprotective agents. ${ }^{16)}$ In a cross-sectional survey of more than 300,000 Americans, only $27.2 \%$ in the group at a high risk for gastropathy took gastroprotective agents. The main reasons for the low administration rate of gastroprotective agent were no GI symptoms, additional cost, and too many drugs to take simultaneously. ${ }^{17)}$

Selective COX-2 inhibitors were developed to reduce GI toxicity. However, Cox-2 inhibitors are not GI risk-free and have increased ulcer risk with long-term use; 
Park and Kwon. Naproxen/Esomeprazole Combination Drug

Clinics in Orthopedic Surgery • Vol. 12, No. 1, $2020 \bullet$ www.ecios.org

Table 4. Analysis the Adherence by the Stratified Demographic and Medical Information

\begin{tabular}{|c|c|c|}
\hline Variable & MMAS & $p$-value \\
\hline Compliance & $6.38 \pm 1.77$ & \\
\hline
\end{tabular}

\begin{tabular}{|c|c|c|}
\hline \multicolumn{3}{|l|}{ Factor } \\
\hline \multicolumn{3}{|l|}{ Sex } \\
\hline Male & $6.46 \pm 1.70$ & \\
\hline Female & $6.30 \pm 1.83$ & \\
\hline \multicolumn{3}{|l|}{ Age (yr) } \\
\hline $19-29$ & $5.12 \pm 1.67$ & \\
\hline $30-39$ & $5.90 \pm 1.72$ & \\
\hline $40-49$ & $6.04 \pm 1.83$ & \\
\hline 50-59 & $6.27 \pm 1.77$ & \\
\hline $60-69$ & $6.38 \pm 1.74$ & \\
\hline$\geq 70$ & $6.58 \pm 1.76$ & $<0.001$ \\
\hline
\end{tabular}

\section{Drinking}

$\begin{array}{lll}\text { Current drinker } & 6.29 \pm 1.74 & \\ \text { Ex-drinker } & 6.27 \pm 1.83 & \\ \text { Non-drinker } & 6.44 \pm 1.78 & <0.001\end{array}$

Smoking

\begin{tabular}{|lll}
\hline Current smoker & $6.43 \pm 1.65$ & 0.006 \\
\hline Ex-smoker & $6.33 \pm 1.73$ & \\
\hline Non-smoker & $6.38 \pm 1.80$ & \\
\hline Duration of disease & & \\
\hline$\geq$ Mean & $6.26 \pm 1.79$ & $<0.001$ \\
\hline$<$ Mean & $6.40 \pm 1.78$ & $<0.001$ \\
\hline Arthritis type & \\
\hline Osteoarthritis & $6.40 \pm 1.78$ & \\
\hline Rheumatoid arthritis & $6.05 \pm 1.61$ & \\
\hline Ankylosing spondylitis & $6.17 \pm 1.55$ & $<0.001$ \\
\hline Comorbidity & \\
\hline Yes & $6.39 \pm 1.80$ & \\
\hline No & $6.37 \pm 1.74$ & \\
\hline Body mass index & $6.24 \pm 1.82$ & \\
\hline$\geq$ Median & $6.34 \pm 1.75$ & \\
\hline$<$ Median & & \\
\hline
\end{tabular}

Values are presented as mean \pm standard deviation. MMAS: Morisky Medication Adherence Scale. therefore, concurrent administration of gastroprotective agents are recommended for patients with a high risk of GI troubles. ${ }^{18)}$ Moreover, concerns regarding the risks of $\mathrm{CV}$ complications have been raised by inhibition of COX-2derived antithrombotic and antiplatelet activity. Rofecoxib was found to have a significantly increased risk for thrombotic events including myocardial infarction and has been banned in the market. ${ }^{19)}$ With respect to diclofenac and ibuprofen, the $\mathrm{CV}$ complication risk was reported to be similar to that of selective COX-2 inhibitors when used in high dosage over a long term; however, naproxen did not have similar risks at a high dose. ${ }^{6}$ The International Working Party and the American College of Gastroenterology also recommend naproxen as the primary therapy for patients at high risk for GI and CV side effects. ${ }^{4,20}$ Moreover, two well-designed, 12-week studies showed that the combination drug was not inferior to celecoxib in treating the signs and symptoms of arthritis and had a significantly low incidence of gastric ulcers. ${ }^{7,21)}$

From the results of this study, coxibs were the most commonly prescribed drug class before the switch to the combination drug. The increased risk of CV complications by coxibs can be greater than $20 \%$ and the mortality and sequelae of $\mathrm{CV}$ complications should be considered when prescribing them to high-risk patients. ${ }^{22)}$ The rate of taking gastroprotective agents was $57 \%$, which is higher than that observed in a previous domestic study; however, the actual adherence may be lower. $\mathrm{H} 2$ blockers were the most prescribed agent, but PPIs have been proven to be more effective. Changing to combination drug can improve the patient adherence to potent PPIs. However, long-term use of PPI may induce a wide range of adverse effects, such as increased risk of infection, reduced intestinal absorption of vitamins and minerals, kidney damage, dementia and osteoporosis. So, more concerns for complications regarding long-term prescription should be given to elderly patients with significant comorbidities. ${ }^{23)}$

The highest improvement of QOL was observed when changing to the combination drug was due to the burden of cost. This means that not only clinical concerns, such as safety and efficacy, but also realistic factors related to real life should be considered. A comparative study of cost-effectiveness between selective COX-2 inhibitors and traditional NSAIDs with PPIs indicated that they are almost equivalent and thus drug selection should be focused on minimizing adverse effects. ${ }^{24)}$ The patients who did not receive a gastroprotective agent previously had a higher QOL improvement. This is consistent with the result in previous studies suggesting that GI trouble is one of the greatest risk factors for QOL decrease. ${ }^{25)}$ 


\section{Table 5. Analysis the Satisfaction by the Stratified Demographic and Medical Information}

\begin{tabular}{|c|c|c|c|c|}
\hline Variable & Satisfactory & Unsatisfied & No difference & $p$-value \\
\hline Satisfaction & $1,338(62.91)$ & 270 (12.69) & $517(24.31)$ & \\
\hline \multicolumn{5}{|l|}{ Factor } \\
\hline \multicolumn{5}{|l|}{ Sex } \\
\hline Male & $626(60.72)$ & $118(11.45)$ & $287(27.84)$ & \\
\hline Female & 712 (65.08) & 152 (13.89) & $230(21.02)$ & $<0.001$ \\
\hline \multicolumn{5}{|l|}{ Age (yr) } \\
\hline $19-29$ & 19 (63.33) & $1(3.33)$ & $10(33.33)$ & \\
\hline 30-39 & 42 (67.74) & $7(11.29)$ & $13(20.97)$ & \\
\hline $40-49$ & $98(64.47)$ & $19(12.5)$ & 35 (23.03) & \\
\hline $50-59$ & $286(71.50)$ & $41(10.25)$ & $73(18.25)$ & 0.014 \\
\hline $60-69$ & $429(61.11)$ & $99(14.10)$ & 174 (24.79) & \\
\hline$\geq 70$ & $464(59.56)$ & 103 (13.22) & 212 (27.21) & \\
\hline \multicolumn{5}{|l|}{ Drinking } \\
\hline Current drinker & $366(63.10)$ & $67(11.55)$ & 147 (25.34) & \\
\hline Ex-drinker & $140(71.07)$ & $24(12.18)$ & 33 (16.75) & \\
\hline Non-drinker & 793 (61.38) & 175 (13.54) & 324 (25.08) & \\
\hline \multicolumn{5}{|l|}{ Smoking } \\
\hline Current smoker & $198(65.13)$ & $30(9.87)$ & $76(25.00)$ & \\
\hline Ex-smoker & $168(70.00)$ & $27(11.25)$ & 45 (18.75) & 0.047 \\
\hline Non-smoker & 931 (61.25) & 207 (13.62) & 382 (25.13) & \\
\hline \multicolumn{5}{|l|}{ Duration of disease } \\
\hline$\geq$ Mean & 356 (54.94) & $121(18.67)$ & 171 (26.39) & \\
\hline$<$ Mean & 898 (66.32) & $134(9.90)$ & 322 (23.78) & $<0.001$ \\
\hline \multicolumn{5}{|l|}{ Arthritis type } \\
\hline Osteoarthritis & 1,268 (63.75) & 256 (12.87) & 465 (23.38) & 0.003 \\
\hline Rheumatoid arthritis & $40(52.63)$ & $9(11.84)$ & $27(35.53)$ & \\
\hline Ankylosing spondylitis & $30(50.00)$ & $5(8.33)$ & $25(41.67)$ & \\
\hline \multicolumn{5}{|l|}{ Comorbidity } \\
\hline Yes & 639 (55.04) & $163(14.04)$ & 359 (30.92) & \\
\hline No & 699 (72.51) & $107(11.10)$ & 158 (16.39) & $<0.001$ \\
\hline \multicolumn{5}{|l|}{ Body mass index } \\
\hline$\geq$ Median & $582(64.03)$ & 126 (13.86) & 201 (22.11) & \\
\hline$<$ Median & 595 (66.04) & $129(14.32)$ & $177(19.64)$ & \\
\hline
\end{tabular}

Values are presented as number (\%). 
One of the most important factors for successful treatment of arthritic conditions is to increase adherence. Medium adherence can be observed when a combination drug is used, and special attention should be paid in patients with poor adherence. In addition, particular care should be taken with patients who are younger than 70 years and have a history of alcohol consumption, a long disease duration, and comorbidities.

With respect to satisfaction, satisfactory was the most common response but overall satisfaction was decreased in elderly patients, those with a long disease duration, and with comorbidities. This suggests that it is difficult to obtain high satisfaction in the long-term treatment of chronic arthritis in elderly patients and additional considerations are needed to improve overall satisfaction. The incidence and severity of adverse events after the drug change were not significantly different from those after general NSAID administration. There were no serious adverse events that clearly demonstrated a causal relationship with drug change. The main limitation of this study is that the observation period was not long enough to accurately assess QOL, satisfaction, and compliance after drug change.

The most important aspects of drug treatment in patients with chronic arthritis are to minimize adverse drug reactions and to improve overall QOL. The choice of NSAID should be tailored to the GI and CV risk in each patient. In this study, the combination drug improved overall QOL and satisfaction, and sex, age, drinking, smoking, disease duration, comorbidities, and BMI might be associated with satisfaction and adherence. We believe that the results of this study can be useful for the prescription of NSAIDs in the treatment of patients with arthritis and can help to predict prognosis after a drug change.

\section{CONFLICT OF INTEREST}

No potential conflict of interest relevant to this article was reported.

\section{ACKNOWLEDGEMENTS}

This study was supported by research grants from Hanmi Pharmaceutical Co., Ltd.

\section{REFERENCES}

1. Paulose-Ram R, Hirsch R, Dillon C, Gu Q. Frequent monthly use of selected non-prescription and prescription nonnarcotic analgesics among U.S. adults. Pharmacoepidemiol Drug Saf. 2005;14(4):257-66.

2. Lanas A, Ferrandez A. Inappropriate prevention of NSAIDinduced gastrointestinal events among long-term users in the elderly. Drugs Aging. 2007;24(2):121-31.

3. Smith SR, Deshpande BR, Collins JE, Katz JN, Losina E. Comparative pain reduction of oral non-steroidal anti-inflammatory drugs and opioids for knee osteoarthritis: systematic analytic review. Osteoarthritis Cartilage. 2016;24(6):96272.

4. Korean Knee Society Subcommittee on Osteoarthritis Guidelines. Guidelines for the treatment of osteoarthritis of the knee. J Korean Knee Soc. 2010;22(1):69-74.

5. Lanza FL, Chan FK, Quigley EM; Practice Parameters Committee of the American College of Gastroenterology. Guidelines for prevention of NSAID-related ulcer complications. Am J Gastroenterol. 2009;104(3):728-38.

6. Kearney PM, Baigent C, Godwin J, Halls H, Emberson JR, Patrono C. Do selective cyclo-oxygenase-2 inhibitors and traditional non-steroidal anti-inflammatory drugs increase the risk of atherothrombosis? Meta-analysis of randomised trials. BMJ. 2006;332(7553):1302-8.
7. Datto C, Hellmund R, Siddiqui MK. Efficacy and tolerability of naproxen/esomeprazole magnesium tablets compared with non-specific NSAIDs and COX-2 inhibitors: a systematic review and network analyses. Open Access Rheumatol. 2013;5:1-19.

8. La Montagna G, Tirri G, Cacace E, et al. Quality of life assessment during six months of NSAID treatment [Gonarthrosis and Quality of Life (GOAL) Study]. Clin Exp Rheumatol. 1998;16(1):49-54.

9. Fagnani F, Bouvenot G, Valat JP, et al. Medico-economic analysis of diacerein with or without standard therapy in the treatment of osteoarthritis. Pharmacoeconomics. 1998;13(1 Pt 2):135-46.

10. Gabriel SE, Campion ME, O'Fallon WM. Patient preferences for nonsteroidal antiinflammatory drug related gastrointestinal complications and their prophylaxis. J Rheumatol. 1993;20(2):358-61.

11. Rabin R, de Charro F. EQ-5D: a measure of health status from the EuroQol Group. Ann Med. 2001;33(5):337-43.

12. Norman GR, Sloan JA, Wyrwich KW. Interpretation of changes in health-related quality of life: the remarkable universality of half a standard deviation. Med Care. 2003;41(5):582-92.

13. Morisky DE, Ang A, Krousel-Wood M, Ward HJ. Predictive 
validity of a medication adherence measure in an outpatient setting. J Clin Hypertens (Greenwich). 2008;10(5):348-54.

14. Laine L, Connors L, Griffin MR, Curtis SP, Kaur A, Cannon CP. Prescription rates of protective co-therapy for NSAID users at high GI risk and results of attempts to improve adherence to guidelines. Aliment Pharmacol Ther. 2009;30(7):767-74.

15. Kim HC, Lee MC, Moon YW, et al. The pattern of use of oral NSAIDs with or without co-prescription of gastroprotective agent for arthritic knee by Korean practitioners. Knee Surg Relat Res. 2011;23(4):203-7.

16. Sturkenboom MC, Burke TA, Dieleman JP, Tangelder MJ, Lee F, Goldstein JL. Underutilization of preventive strategies in patients receiving NSAIDs. Rheumatology (Oxford). 2003;42 Suppl 3:iii23-31.

17. Abraham NS, El-Serag HB, Johnson ML, et al. National adherence to evidence-based guidelines for the prescription of nonsteroidal anti-inflammatory drugs. Gastroenterology. 2005;129(4):1171-8.

18. Stockl K, Cyprien L, Chang EY. Gastrointestinal bleeding rates among managed care patients newly started on cox2 inhibitors or nonselective NSAIDs. J Manag Care Pharm. 2005;11(7):550-8.

19. Warner TD, Mitchell JA. COX-2 selectivity alone does not define the cardiovascular risks associated with non-steroidal anti-inflammatory drugs. Lancet. 2008;371(9608):270-3.

20. Chan FK, Abraham NS, Scheiman JM, Laine L; First Inter- national Working Party on Gastrointestinal and Cardiovascular Effects of Nonsteroidal Anti-inflammatory Drugs and Anti-platelet Agents. Management of patients on nonsteroidal anti-inflammatory drugs: a clinical practice recommendation from the First International Working Party on Gastrointestinal and Cardiovascular Effects of Nonsteroidal Anti-inflammatory Drugs and Anti-platelet Agents. Am J Gastroenterol. 2008;103(11):2908-18.

21. Hochberg MC, Fort JG, Svensson O, Hwang C, Sostek M. Fixed-dose combination of enteric-coated naproxen and immediate-release esomeprazole has comparable efficacy to celecoxib for knee osteoarthritis: two randomized trials. Curr Med Res Opin. 2011;27(6):1243-53.

22. Scheiman JM, Hindley CE. Strategies to optimize treatment with NSAIDs in patients at risk for gastrointestinal and cardiovascular adverse events. Clin Ther. 2010;32(4):667-77.

23. Eusebi LH, Rabitti S, Artesiani ML, et al. Proton pump inhibitors: risks of long-term use. J Gastroenterol Hepatol. 2017;32(7):1295-302.

24. Latimer N, Lord J, Grant RL, et al. Cost effectiveness of COX 2 selective inhibitors and traditional NSAIDs alone or in combination with a proton pump inhibitor for people with osteoarthritis. BMJ. 2009;339:b2538.

25. Yeomans N, Wilson I, Langstrom G, et al. Quality of life in chronic NSAID users: a comparison of the effect of omeprazole and misoprostol. Scand J Rheumatol. 2001;30(6):32834. 